\title{
Modelling of low-velocity impacts on composite beams in large displacement
}

\author{
M. Utzeri*, M. Sasso \\ Università Politecnica delle Marche - Department of Industrial Engineering and Mathematics, Italy \\ m.utzeri@pm.univpm.it,m.sasso@univpm.it \\ G. Chiappini \\ Università degli Studi eCampus, Italy \\ gianluca.chiappini@uniecampus.it \\ S. Lenci \\ Università Politecnica delle Marche - Department of Civil and Building Engineering, and Architecture, Italy \\ s.lenci@univpm.it
}

\begin{abstract}
The paper provides an evaluation of the nonlinear dynamic response of a cantilever beam made of composite material subjected to low-velocity impacts. The structure is assumed to respond in a quasi-static manner and modelled by a continuous beam in large displacement with a lumped mass attached. First, an analytical model was developed to study the free vibrations of a beam, taking into account the nonlinearities due to large displacements and inertia. Then, the analytical findings were compared with experimental test data. The vibration of a real composite beam has been acquired through high-speed imaging technique. The displacements of the beam were extracted by digital image analysis; then, the nonlinear parameters of the analytical model were determined by the Fitting Time History technique. The results obtained by the analytical model and the experimental test are compared with numerical analysis. The validated analytical model was adapted to study a low-velocity impact; the lumped mass was associated with a rigid projectile, whose initial speed represents the impact velocity.
\end{abstract}

KEYwORDS. Impact Dynamics; Composite Beam; Nonlinear Frequencies; Nonlinear Dynamics.

\section{OPEN $\bigcirc$ ACCESS}

Citation: Utzeri, M, Sasso, M., Chiappini, G., Lenci, S.., Modelling of low-velocity impacts on composite beams in large displacement, Frattura ed Integrità Strutturale, 58 (2021) 254-271.

Received: 18.08 .2021

Accepted: 01.09.2021

Published: 01.10.2021

Copyright: (C) 2021 This is an open access article under the terms of the CC-BY 4.0, which permits unrestricted use, distribution, and reproduction in any medium, provided the original author and source are credited.

\section{INTRODUCTION}

$\mathrm{I}$ $\mathrm{n}$ the last few years, composite materials (e.g. Carbon Fiber Reinforced Polymer, Glass Fiber Reinforced Polymer) are used extensively in widely impact engineering applications. The mains composite materials peculiarities are the high specific strength and flexibility. Therefore, a composite structure can undergo large deflection without breaks when a foreign object hits it. The theory behind the low-velocity impacts has been hugely discussed by Abrate with several works 
on this topic $[1,2]$. The aim of his work is to extend the known model to predict the contact force history and the overall response of the structure in large displacements. The relevant models used to study the low-velocity impact are spring-mass and energy balance models [3]. In this study the spring-mass approach was used to describe the overall response of a slender cantilever beam which undergoes the impact of a projectile. Starting from the Abrate theory, the spring associated with the structure is analytically modelled as a continuous Euler-Bernoulli beam. The indentation phenomenon was neglected because the overall deflection of the beam is much larger than local. In this way, the mass of projectile can be added to the continuous cantilever beam. However, the slender beam reaches large deflection to absorb the projectile energy during the impact so only the nonlinear vibration theory allows establishing the correct dynamical behaviour. Many theoretical and experimental investigations of nonlinear vibrations of beams have appeared over the years $[4,5,6,7]$. In general, the nonlinearity may be attributed to geometry (stiffness and inertia), and material (constitutive equation and damping) [8]. Firstly, geometric nonlinearities are caused by large displacements and slopes. Consequently, it is not possible to use the small-angle assumption, which would reduce the nonlinear curvature to the simple linear form [9, 10, 11]. For a deeper discussion on the nonlinear curvature the reader is referred to $[12,13]$. Nonlinear inertia effects may be caused by the presence of concentrated or distributed masses. Actually, for transversal vibrations of an initially straight beam there are transversal and axial (linear and nonlinear) inertial effects; while the former can never be neglected, in certain cases (for example for axially immovable boundary conditions), the latter are negligible [5, 14]. According to Hamdan [15, 16, 17], the nonlinear stiffness induces nonlinear features of the hardening type, i.e., the vibration frequencies increase by increasing the amplitude of motion; on the contrary, the inertial nonlinearity, which is related to the inextensibility condition and to the lumped mass, determines a softening behaviour of the so-called "backbone curve". Consequently, the nonlinear effects which modify the frequencies act also on the time in which the impact occurs. Therefore, the impact time is strictly connected with the amplitude reached during the impact. For instance, a hardening behaviour on the nonlinear frequency clearly reduces the impact time and the maximum amplitude reaches a lower amplitude than linear form. Accordingly, in this paper, the effects of nonlinearity in a cantilever beam were addressed further elaborating and extending the analytical approach of the works $[16,15,18]$. Analytical model takes into account the nonlinearity derived by large amplitude vibration and inertia. Starting from the energy definition, the equation of motion is determined including nonlinear terms associated to geometrical nonlinearity up to the fifth order. Hence, enforcing stationarity of the lagrangian of the system yields to the unimodal equation, which can be solved by Multiple Scale Method [19]. This analytical approach gives the approximate solution of the transversal displacement and the first nonlinear frequency where the nonlinear terms are up to fifth order. Then, the results of an experimental tests were compared with the the analytical findings to validate the nonlinear model. Free vibration experimental tests were done on a rectangular beam made of twill laminated carbon fiber reinforced polymer, whose vibration displacement amplitude was measured by high-speed imaging; indeed, the image analysis allows measuring the vibration displacement amplitude of any point of the beam. The obtained results were post-processed by the Fitting Time History (FTH) technique, which is based on the least square approximation of general damped sinusoidal function with the measured free damped vibrations of the specimen. This technique allows to determine the main natural frequencies, the related modal damping coefficients, and damping and frequency nonlinearities [20, 21]. Finally, the projectile impact against a cantilevered beam has been simulated through the commercial Finite Element software Abaqus (C). Exploiting the nonlinear Euler-Bernoulli beam coupled with implicit solver, the data about speed and displacement were exported and compared with the analytical findings.

\section{ANALYTICAL MODEL}

I $\mathrm{n}$ according to Abrate [3], impacts can be modelled through spring-mass models. They provide accurate solution for low-velocity impacts. The most complete model consist in two-degree of freedom model which the projectile and the hit beam are associated with masses. The spring links each other represents the nonlinear contact stiffness. Instead, the second spring is associated with the linear and nonlinear stiffness of the structure. This kind of model can be exploited to describe the impact dynamics of projectile against a slender beam. The stiffness of the structure can be associated with the bending stiffness of Euler-Bernoulli beam. However, the hit beam undergoes a large deflection because of the slenderness. Therefore, the indentation is smaller than the overall deformation of the structure and can be neglected. The models assumes the single-degree of freedom form which the masses of the projectile and the beam are added together, as shown in Fig. (2). The low-velocity impacts models exist only when the waves phenomena inside the beam due to the hitting are neglected. In this case, flexural waves appear from the bending action of the impacted projectile. The Euler-Bernoulli theory of bending assumes that plane sections remain plane as the bending deformation. Shear deformation and rotary 
inertia effects are ignored. Considering a uniform beam of mass per unit length $\mathrm{m}$, the elastic modulus $\mathrm{E}$ and the area moment of inertia I that undergoes a flexural wave motion with vertical displacement, the flexural waves speed is given by

$$
c_{F}=\frac{\omega}{\gamma}=\left(\frac{E I}{m}\right)^{\frac{1}{4}} \sqrt{\omega}
$$

where $\gamma$ is the wavenumber and $\omega$ is the frequency $[22,23]$. From the Eqn. (1) can be deduce the flexural waves speed is function of frequency thus they are also called dispersive waves. The phenomenon of flexural wave propagation can be neglected when the interested time is bigger than the time of flexural wave takes to travel inside the beam, $t_{F W}=c_{F} L$. Fig. (1) shows a simplified motion of slender cantilever beam during an impact of projectile. When the projectile hit the beam Fig. (1b), flexural waves start to travel inside the beam and have a velocity $c_{F} \cdot c_{F}$ is not constant as in axial waves but it is depended to the impact. More rapidly is the impulse and more is the waves speed. After the early stages, multiple reflections occur, and the bending motion is established. As the beam is bending, flexural waves keep traveling but their effects can be neglected. Consequently, the beam motion can be completely described through multi-modal superposition technique as in Fig. (1c).

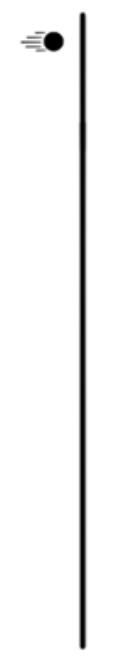

(a)

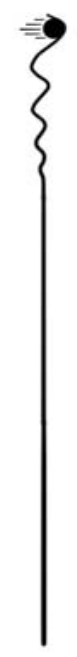

(b)

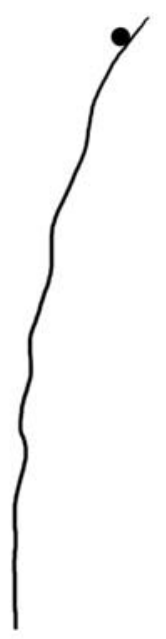

(c)

Figure 1: Simplified motion of slender cantilever beam during an impact of projectile. (a) Before the impact. (b) After the impact and $t<t_{F W}$ (Flexural waves predominant). (c)After the impact and $t \gg t_{F W}$ (Multi-modal superposition)

\section{Multi-Modal Nonlinear Response}

The beam under consideration is shown in Fig. (2). It is homogeneous, i.e. the cross-section, the density, and all other properties are constant along the beam axis. The lumped mass is fixed symmetrically with respect to the beam center line to maintain the symmetry with respect to the $X$ reference line.

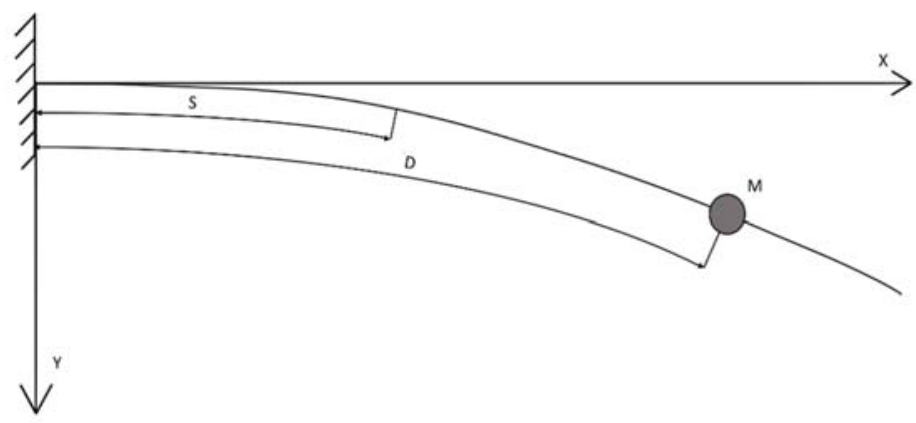

Figure 2: Sketch of cantilever beam with intermediate mass 
In order to describe the nonlinear response of the impacted beam in large deflection, the equations of motion are determined starting from the definition of the energies. The formulation takes into account large vibrations, the inextensibility of the beam, and the lumped mass inertial terms. As proposed by Hamdan [17], the kinetic $\left(E_{k}\right)$ and potential $\left(E_{p}\right)$ energy of the beam are defined as

$$
\begin{aligned}
& E_{k}=\frac{m L^{3}}{2}\left[\int_{0}^{1}\left|\left(\dot{x}^{2}+\dot{y}^{2}\right) d \zeta+\mu\left(\dot{x}^{2}+\dot{y}^{2}\right)_{\eta}+j \dot{\theta}^{2}\right|_{\eta}\right] \\
& E_{p}=\frac{E I}{2 L} \int_{0}^{1}(k \zeta, t)^{2} d \zeta
\end{aligned}
$$

where dot means derivative with respect to time, $\zeta=\frac{S}{L}$ is the dimensionless distance from the left boundary, $x=\frac{X}{L}$ and $y=\frac{Y}{L} \cdot X(\zeta, t)$ and $Y(\zeta, t)$ are the deformed position of the generic point $(\zeta, 0)$ in the reference straight configuration. $\eta=\frac{D}{L}$ is the given dimensionless position of the lumped mass. $\mu=\frac{M}{m L}$ and $j=\frac{J}{m L^{3}}$ are the inertia dimensionless parameters of the lumped mass. The term $k(\zeta, t)$ is the curvature of the neutral axis of the beam, and is given by:

$$
k=\theta^{\prime}
$$

Here prime means derivative with respect to $\zeta$, and $\theta$ is the rotation (equal to the beam slope, since we are considering an Euler-Bernoulli beam) [24],

$$
\tan (\theta)=\frac{y^{\prime}}{x^{\prime}} \tan (\theta)^{\prime}=\frac{1}{\cos (\theta)^{2}} \theta^{\prime} \quad \theta^{\prime}=\frac{y^{\prime \prime} x^{\prime}-y^{\prime} x^{\prime \prime}}{x^{\prime 2}+y^{\prime 2}}
$$

In this work, as in [6], the axis of the beam is considered inextensible. This implies that:

$$
x^{\prime 2}+y^{\prime 2}=1
$$

namely,

$$
x^{\prime}=\sqrt{\left(1-y^{\prime 2}\right)}=1-\frac{y^{\prime 2}}{2}-\frac{y^{\prime 4}}{8}-\frac{y^{\prime 6}}{16}+\ldots
$$

where in the last expression a Taylor approximation is considered. By means of Eqn.(7) we obtain, up to the due order,

$$
\begin{aligned}
& \theta=y^{\prime}+\frac{y^{\prime 3}}{6}+\frac{3 y^{15}}{40}+\ldots \\
& \dot{\theta}^{2}=\dot{y}^{\prime 2}\left(1+y^{\prime 2}+y^{\prime 4}+\ldots\right)
\end{aligned}
$$

(use is made of the boundary condition $x(0)=0$ )

$$
k=\frac{y^{\prime \prime}}{\sqrt{1-y^{\prime 2}}}=y^{\prime \prime}\left(1+\frac{y^{\prime 2}}{2}+\frac{3 y^{\prime 4}}{8}+\ldots\right)
$$


Note that the higher order terms are positive, meaning that the curvature increases (with respect to the linear case) for increasing amplitudes. This is the motivation for the hardening behaviour due to the geometric stiffness nonlinearity. The expression of the kinetic energy of the system then becomes:

$$
\begin{aligned}
E_{k} & =\frac{m L^{3}}{2}\left\{\int_{0}^{1}\left[\dot{y}^{2}+\left(\int_{0}^{\zeta} \dot{y}\left(y^{\prime}+\frac{y^{\prime 3}}{2}+\frac{3 y^{\prime 5}}{8}\right) d \chi\right)^{2}\right] d \zeta\right. \\
& +\mu\left[\dot{y}^{2}+\left(\int_{0}^{\zeta} \dot{y}\left(y^{\prime}+\frac{y^{13}}{2}+\frac{3 y^{\prime 5}}{8}\right) d \chi\right)^{2}\right]_{\eta} \\
& \left.+j\left[\dot{y}^{\prime 2}\left(1+y^{\prime 2}+y^{\prime 4}\right)\right]_{\eta}\right\}
\end{aligned}
$$

Likewise, the potential energy becomes:

$$
E_{p}=\frac{E I}{2 L} \int_{0}^{1} y^{\prime 2}\left(1+\frac{y^{\prime 2}}{2}+\frac{3 y^{\prime 4}}{8}\right)^{2} d \zeta
$$

The vibration motion is assumed to have linear modes. The vibration of the beam with a concentrate intermediate mass is governed by the equation

$$
y^{\prime \prime \prime+}+\frac{m}{E I}(1+\mu \delta(\zeta-\eta)) \stackrel{y}{y}=0
$$

Assuming a solution of Eqn.(13) in the form

$$
y(\zeta, t)=\Phi(\zeta) e^{i \omega t}
$$

Substituting the Eqn.(14) into Eqn.(13) gives

$$
\Phi(\zeta)^{\prime \prime \prime}-\frac{m \omega^{2}}{E I}(1+\mu \delta(\zeta-\eta) \Phi(\zeta))=0
$$

and the boundary condition at $x=0$ and $x=1$ are

$$
\Phi(0)=0 \quad \Phi(0)^{\prime}=0 \quad E I \Phi(1)^{\prime \prime}=0 \quad E I \Phi(1)^{\prime \prime \prime}=0
$$

Taking the Laplace transformation of Eqn. (15), using the boundary conditions (16) and then taking the inverse Laplace transformation yields the eigenfunction of the beam with an intermediate concentrated mass

$$
\begin{aligned}
\Phi(\zeta) & =\frac{\Phi(0) "}{2} k^{2}(\cosh (\zeta k L)-\cos (\zeta k L))+\frac{\Phi(0) " '}{2} k^{3}(\sinh (\zeta k L)-\sin (\zeta k L)) \\
& +\frac{\Phi(\eta)}{2} k L^{2} \mu H(\zeta-\eta)(\sinh (k L(\zeta-\eta))-\sin (k L(\zeta-\eta)))
\end{aligned}
$$

where $k^{4}=\frac{m \omega^{2}}{E I}$. Hence the unknown constants $\Phi "_{0}$ and $\Phi "_{0}$ can be determined imposing the boundary condition in Eqn.(17). Substituting $\Phi "_{0}$ and $\Phi "_{0}{ }_{0}$ into Eqn. (17), and then letting $\zeta=\eta$ to eliminate $\Phi(\eta)$, gives the characteristic equation 


$$
\begin{aligned}
& 1+\cos (k L) \cosh (k L)+ \\
& \frac{k L \mu}{2}((\cos (k L \eta) \sinh (k L \eta)-\cosh (k L \eta) \sin (k L \eta))(\cos (b k) \cosh (b k)+1)+ \\
& (\cos (b k) \sinh (b k)-\cosh (b k) \sin (b k))(\cos (k L \eta) \cosh (k L \eta)-1))=0
\end{aligned}
$$

where $b=L(1-\eta)$ [25]. Note that the $\Phi(\eta)$ guarantees that $\max |\Phi(\zeta)|=1$. The Eqn.(18) can be simplified assuming $\mu=0$ to obtain the characteristic equation of clamped-free beam case

$$
\cosh (k L) \cosh (k L)+1=0
$$

We consider that the arbitrary motion of a beam undergoing free vibration may be expressed as a superposition of its free vibration mode shapes $\left(\Phi_{n}(\zeta)\right)$, each undergoing simple harmonic motion with frequency $\omega_{n 0}$, namely we assume that:

$$
y(\zeta, t)=\sum_{n=1}^{\infty} u_{n}(t) \Phi_{n}(\zeta)
$$

where $\Phi_{n}(\zeta)$ is a given function and $u(t)$ is the unknown. The lagrangian of the system is defined as $\mathcal{L}=E_{k}-E_{p}$. Inserting the Eqn.(20) in $\mathcal{L}$ and enforcing stationariety, yields to the equation of motion of each mode defined as $\frac{\partial \mathcal{L}}{\partial t \dot{y}_{n}}-$ $\frac{\partial \mathcal{L}}{\partial y_{n}}$. After some computations we get $[16,26]$ :

$$
\ddot{u}_{n}\left(\alpha_{1}+\alpha_{2} u_{n}^{2}+\alpha_{3} u_{n}^{4}\right)+\dot{u}_{n}^{2}\left(\alpha_{2} u_{n}+2 \alpha_{3} u_{n}^{3}\right)+\beta^{2}\left(\alpha_{4} u_{n}+2 \alpha_{5} u_{n}^{3}+3 \alpha_{6} u_{n}^{5}\right)=0
$$

where

$$
\begin{aligned}
\alpha_{1} & =\int_{0}^{1} \Phi_{n}^{2}(\zeta) d \zeta+\mu \Phi_{n}(\eta)^{2}+j \Phi_{n^{\prime}}(\eta)^{2} \\
\alpha_{2} & =\int_{0}^{1}\left(\int_{0}^{\zeta} \Phi_{n^{\prime}}^{2}(\chi) d \chi\right)^{2} d \zeta+\mu\left(\int_{0}^{\eta} \Phi_{n^{\prime}}^{2}(\chi) d \chi\right)^{2}+j \Phi_{n^{\prime}}(\eta)^{4} \\
\alpha_{3} & =\int_{0}^{1}\left(\int_{0}^{\zeta} \Phi_{n^{\prime}}^{2}(\chi) d \chi\right)\left(\int_{0}^{\zeta} \Phi_{n^{\prime}}^{4}(\chi) d \chi\right) d \zeta \\
& +\mu\left(\int_{0}^{\eta} \Phi_{n^{\prime}}^{2}(\chi) d \chi\right)\left(\int_{0}^{\eta} \Phi_{n^{\prime}}^{4}(\chi) d \chi\right)+j \Phi_{n^{\prime}}(\eta)^{6} \\
\alpha_{4} & =\int_{0}^{1} \Phi_{n^{\prime \prime}}^{2}(\zeta) d \zeta \\
\alpha_{5} & =\int_{0}^{1} \Phi_{n^{\prime \prime}}^{2}(\zeta) \Phi_{n^{\prime}}^{2}(\zeta) d \zeta \\
\alpha_{6} & =\int_{0}^{1} \Phi_{n^{\prime \prime}}^{2}(\zeta) \Phi_{n^{\prime}}^{4}(\zeta) d \zeta \\
\beta^{2} & =\frac{E I}{m L^{4}}
\end{aligned}
$$

The nonlinear inertial terms are clearly visible. The accuracy of the proposed approximate solution is expected to diminish when the mass ratio $\mu$ exceeds the value of $3[16]$, since the spatial shapes $\Phi_{n}(\zeta)$ is only considered in linear form. The Eqn.(21) is solved by the Multiple Scale Method [19], and the approximate solution is given by

$$
u_{n}(t)=A_{n} \sin \left(\omega_{n l} t+\phi_{n 1}\right)+\frac{1}{16}\left(\frac{\alpha_{5}}{\alpha_{4}}-\frac{\alpha_{2}}{\alpha_{1}}\right) A_{n}^{3} \sin \left[3\left(\omega_{n l} t+\phi_{n 2}\right)\right]+\ldots
$$

The $\omega_{n l}$ is the nonlinear frequency, and can be written in the form

$$
\omega_{n l}=\omega_{n 0}+\omega_{n 2} A_{n}^{2}+\omega_{n 4} A_{n}^{4}+\ldots
$$


where:

$$
\begin{aligned}
& \omega_{n 0}=\beta \sqrt{\frac{\alpha_{4}}{\alpha_{1}}}, \\
& \omega_{n 2}=\frac{\omega_{n 0}}{4}\left(3 \frac{\alpha_{5}}{\alpha_{4}}-\frac{\alpha_{2}}{\alpha_{1}}\right), \\
& \omega_{n 4}=\frac{\omega_{n 0}}{64}\left(-15 \frac{\alpha_{5}^{2}}{\alpha_{4}^{2}}-18 \frac{\alpha_{2} \alpha_{5}}{\alpha_{1} \alpha_{4}}+9 \frac{\alpha_{2}^{2}}{\alpha_{1}^{2}}+60 \frac{\alpha_{6}}{\alpha_{4}}-12 \frac{\alpha_{3}}{\alpha_{1}}\right)
\end{aligned}
$$

Eqn.(24) is the analytical expression of the backbone curve, approximated up to the fifth order. Considering only the first term in Eqn.(23), we remind that the physical tranvsersal position of the beam, which is needed for comparison with numerical and experimental results, is given by

$$
Y(\zeta, t)=L \sum_{n=1}^{\infty} u_{n}(t) \Phi_{n}(\zeta)=L \sum_{n=1}^{\infty} \mathrm{s} A_{n} \operatorname{in}\left(\omega_{n l} t+\phi_{n}\right) \Phi_{n}(\zeta)
$$

In low-velocity impact dynamics the analytical model provides two boundary condition. The initial displacement of the system is zero. Secondly, the initial velocity of the system coincides with the impact velocity $v_{0}$. In a continuous EuleroBernoulli beams with a intermediate lumped mass the boundary conditions can be imposed through the Dirac function. Thereby the initial velocity along the beam is zero except in the point where the impact occurs.

$$
y(\zeta, 0)=0 \quad \dot{y}(\zeta, 0)=g(\zeta)=v_{0} \delta(\zeta-\eta)
$$

If the impact occurs in a portion of the beam, the $g(\zeta)$ can be described through boxcar function or rectangular function as

$$
g(\zeta)=v_{0} \Pi\left(\frac{\zeta-\eta}{\zeta_{\operatorname{Imp}}}\right)=v_{0}\left(H\left(\zeta-\left(\eta-\frac{\zeta_{\operatorname{Imp}}}{2}\right)\right)-H\left(\zeta-\left(\eta+\frac{\zeta_{\operatorname{Imp}}}{2}\right)\right)\right)
$$

where the $\zeta_{\text {Imp }}$ is the adimensional portion of the beam impacted. Then, substituting the Eqn.(20) in Eqn.(27) yields

$$
\begin{aligned}
& y(\zeta, 0)=\sum_{n=1}^{\infty} \mathrm{s} A_{n} \operatorname{in}\left(\phi_{n 1}\right) \Phi_{n}(\zeta)=0, \\
& \dot{y}(\zeta, 0)=v_{0} \delta(\zeta-\eta)=\sum_{n=1}^{\infty} \mathrm{c} A_{n} \omega_{n} \operatorname{os}\left(\phi_{n 1}\right) \Phi_{n}(\zeta)
\end{aligned}
$$

The initial displaced shape have to be equal to zero so the elementary solution is $\phi_{n 1}=0$. Thus, in this work the $A_{n}$ is only function of impact velocity. The magnitudes represents the strengths in which the various modes participate in the subsequent motion [27]. Multiplying both sides of Eqn.(30) by the $m$-th eigenfunction, integrating both sides over the length of the beam, and taking advantage of the well-known orthogonality property of the eigenfunctions yields to $A_{n}$, the amplitude coefficients of the $n$-th mode

$$
A_{n}=\frac{\int_{0}^{1} g(\zeta) \Phi_{n}(\zeta) d \zeta}{\omega_{n} \int_{0}^{1} \Phi_{n}(\zeta)^{2} d \zeta}=\frac{\int_{0}^{1} v_{0} \delta(\zeta-\eta) \Phi_{n}(\zeta) d \zeta}{\omega_{n} \int_{0}^{1} \Phi_{n}(\zeta)^{2} d \zeta}
$$




\section{EXPERIMENTAL METHOD}

$\mathrm{T}$ he aim of the experimental test is to validate the analytical model proposed in the previous section. The model can be validated through a comparison with the vibration of cantilever beam with an intermediate fixed mass. In this way a more complex experimental test as gas gun test is avoided $[28,29]$. The specimen is subjected to an impulse at its free end and the history of the oscillation amplitude is measured. During beam oscillations, the longitudinal section was recorded by a high speed camera (model FastCam Photron (C) SA4) at frame rate of $10000 \mathrm{~Hz}$. By means of the Matlab Image Processing toolbox, each photo has binarized and processed to obtain the profile of beam longitudinal section, as shown in Fig.3. More detail of the experimental test can be found in Utzeri et al. work [15]. The specimen is a rectangular beam made of carbon fibre reinforced polymer pre-preg T700 TWILL. The linear density of the beam $\mathrm{m}$ is $0.0681 \frac{\mathrm{Kg}}{\mathrm{m}}$. The flexural Young's modulus, i.e. E, are determined experimentally through the standard test method for flexural properties of polymer matrix composite materials (ASTM D7264/D7264M), and it is $44951 \frac{\mathrm{N}}{\mathrm{mm}^{2}}$. The beam cross-section is rectangular, where the base is $25.05 \mathrm{~mm}$ and the height is $1.85 \mathrm{~mm}$.

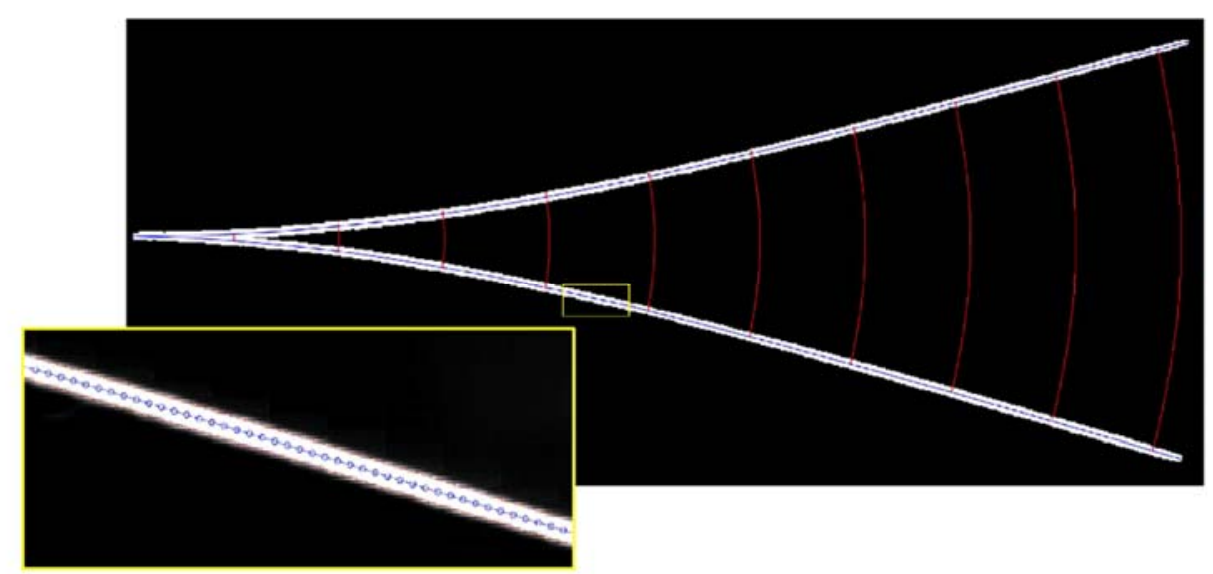

Figure 3: Image processing which shows the transverse vibration of the beam. The blue lines represents the upper and lower profiles of the beam. The circle line and the red line can be associated with the the neutral axis and the trajectory of one beam point, respectively.

\section{NONLINEARITIES IDENTIFICATION}

$\mathrm{F}$

ollowing [20, 21], the results were post-processed by the Fitting Time History (FTH) technique, which is based on the least square approxi-mation of the measured free damped vibrations. FTH technique permits to reveal how the natural frequencies as well as the modal damping coefficients depend nonlinearly on the excitation amplitude in each $i$-th mode. Consequently, this technique is coherent with the analytical method involved in Analytical section, i.e. multimodal approach. According to Eqn.(23), and retaining only the term proportional to A, during the free oscillations the amplitude $q_{i}$ of the $i$-th mode is given by

$$
q(\zeta, t)=L \sum_{n=i}^{\infty} \mathrm{s} A_{i} \text { in }\left(\omega_{i l} t+\phi_{i 0}\right) \Phi_{n}(\zeta)
$$

where $A_{i}(t)=B_{i} e^{-\xi_{i} \omega_{i} t}$ represents the oscillation amplitude, which is assumed to vary in time because of the damping. $\omega_{i l}=\omega_{i} \sqrt{\left(1-\xi_{i}\right)}$ is the damped frequency, $\omega_{i}$ is the natural frequency, $\xi_{i}$ is the damping coefficient and $B_{i}$ and $\phi_{i}$ are the starting amplitude and the phase delay, respectively, that depend on the initial conditions. The linear frequency is initially 
computed by the Fast Fourier Transform (FFT). The nonlinear frequency is a function of the amplitude thus, according to Eqn.(24), we assume:

$$
\omega_{i l}(t)=\omega_{i 0}+\omega_{i 2} A_{i}^{2}(t)+\omega_{i 4} A_{i}^{4}(t)
$$

where $\omega_{i 0}$ is the linear (natural) frequency and $\omega_{i j}$ are the nonlinear correction terms of $j$-th order for the $i$-th mode. Consistently with the above explained approach, the nonlinear damping has been assumed as:

$$
\xi_{i l}(t)=\xi_{i 0}+\xi_{i 1} A_{i}(t)+\xi_{i 2} A_{i}^{2}(t)
$$

FTH optimization needs a cost or error function to be minimized. Therefore, the normal root means square deviation (NRSMD) between analytical function and experimental measures is used:

$$
\operatorname{NRSMD}\left(\xi_{i j}, \omega_{i j}, \phi_{i}, B_{i}\right)=\frac{\sqrt{\frac{\sum_{k=1}^{N}\left(q_{k}-\widehat{y_{k}}\right)^{2}}{N}}}{\max |\hat{y}|}
$$

where $N$ is the number of acquired samples, $\widehat{y_{k}}$ is the $k$-th experimental observation, $q_{k}$ is the value of the analytical function at the same time. The NRSMD provides the optimal estimation of the vibrational properties of the system (linear, $\omega_{i 0}$ and $\xi_{i 0}$, and nonlinear, $\omega_{i 2}, \omega_{i 4}, \xi_{i 1}$ and $\xi_{i 2}$ ). The minimization algorithm was created in-house with Matlab (C). Finally, the nonlinear damping an frequency coefficients were progressively introduced into the Matlab optimization algorithm: first, only linear terms where considered, then a second term was added, and so on. The performance of the optimization algorithm was observed to improve increasingly.

\section{NUMERICAL MODEL}

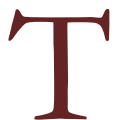
he aim of the numerical modelling is to validate the analytical model proposed in the analytical section. The model can be validated through a comparison with the outcomes obtained by the impact of concentrate mass against the cantilever beam. The FEM analysis was carried out by means ABAQUS (C) software. The elements such as B23, $\mathrm{B} 23 \mathrm{H}, \mathrm{B} 33$, and $\mathrm{B} 33 \mathrm{H}$ allow to model an Euler-Bernoulli beam in ABQUS. So, they do not allow for transverse shear deformation; plane sections initially normal to the beam's axis remain plane (if there is no warping) and normal to the beam axis. In this study, 10000 B23 BEAM was used to model the length of the beam. The concentrate mass is considered rigid with specific initial condition. The mass has a initial speed $v_{0}$ and is located transversely at the beam at $D$ distance from the clamped end. The nonlinear transient analysis coupled with implicit solver has been used to compute the time history of the entire beam deflection. Two load step was used and a very small time step, i.e. $0.00001 \mathrm{~s}$, was imposed in the FEM simulation. The first step defines the initial condition of the beam and the mass. In the second step the condition on the mass speed was deleted. So, the mass can impact against the beam and loose its velocity during the bending motion of the beam. To avoid the indentation effect and rebounds the mass was imposed to be bonded to the beam. After the simulation the data about speed and displacement were exported and compared with the analytical findings.

\section{RESULTS AND DISCUSSIONS}

I

he results obtained by the analytical model were compared with experimental data to validate the nonlinear model. After all, the boundary conditions associated with low-velocity impact were imposed to the model. The analytical findings were discussed and compared to the numerical solutions.

\section{Experimental Validation}

The FTH technique has been applied to the displacement signals measured in the experiment described in Experimental Method Section. A concentrate mass with a mass ratio $\mu=0.5$ was fixed along to the beam at $\eta=0.95$. The vibration response is analyzed through FTH technique. 


\begin{tabular}{ccccc}
\hline & $\begin{array}{c}\text { Unimodal } \\
\text { linear }\end{array}$ & $\begin{array}{c}\text { Unimodal } \\
\text { first order } \\
\text { nonlinearities }\end{array}$ & $\begin{array}{c}\text { Unimodal } \\
\text { second order } \\
\text { nonlinearities }\end{array}$ & Multimodal \\
NRMSD & $4.14 \%$ & $2.64 \%$ & $1.07 \%$ & $0.76 \%$ \\
$\omega_{10}$ & 149.205 & 149.145 & 148.954 & 148.898 \\
$\omega_{12}$ & 0 & 4.125 & 4.815 & 4.232 \\
$\omega_{14}$ & 0 & 0 & 15.5231 & 12.6589 \\
$\xi_{10}$ & 0.0091 & 0.0088 & 0.0063 & 0.0076 \\
$\xi_{11}$ & 0 & 0.1910 & 0.3431 & 0.1793 \\
$\xi_{12}$ & 0 & 0 & -6.0519 & -4.0536 \\
$B_{1}$ & 0.2426 & 0.2433 & 0.2451 & 0.2475 \\
$\phi_{1}$ & 1.3219 & 1.3184 & 1.3170 & 1.2907 \\
$\omega_{20}$ & 0 & 0 & 0 & 1296.5 \\
$\xi_{20}$ & 0 & 0 & 0 & 0.0041 \\
$B_{2}$ & 0 & 0 & 0 & -0.0012 \\
$\phi_{2}$ & 0 & 0 & 0 & 3.0167 \\
\hline
\end{tabular}

Table 1: FTH fitting coefficients for the signal at $\eta=0.95$ of the beam length

The coefficients of the FTH fittings, which synthetize the experimental results and thus are the most important results of this part, are reported in Tab. 1, together with the achieved NRSMD values. It can be noted that, as expected, the NRSMD values reduces as the modeling is enhanced from linear unimodal to multimodal nonlinear. The $\phi_{2}, B_{2}, \phi_{1}, B_{1}$ terms do not have a role in the system characterization. They compare only in the minimization algorithm, and are reported for completeness of information.

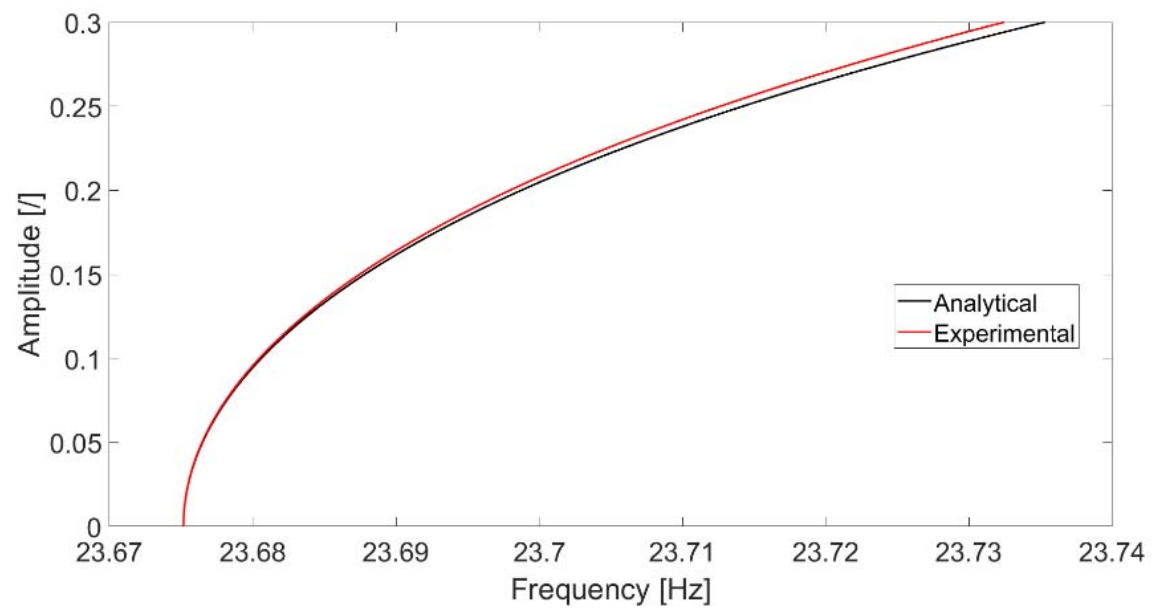

Figure 4: Comparison of backbone curve of the first nonlinear frequency in analytical and experimental results.

It can be noted that the backbone curve obtained by experimental method is very similar to that obtained with the analytical formulation. Moreover, the multi-modal identification increases the performance of the FTH. Indeed, inserting the second mode with its relative nonlinearities, the backbone curve of first frequency approaches that obtained analytically. The analytical model was able to describes correctly the first nonlinear frequency and therefore it was validated. Further information about the efficiency of FTH technique in experimental tests can be found in these works $[15,14]$. 


\section{Analytical Findings}

First of all, the backbone curve of the first three modes are computed by means of Eqn. (24). The Fig. (5) shows the first nonlinear frequency and the influence of impacted distance $\eta$ of a projectile with a mass ratio $\mu$ of $50 \%$ and $200 \%$. In both of cases, the nonlinear behaviour is hardening so the frequency increase with the vibration amplitude. The impacted distance $\eta$ changes the nonlinear hardening trend. Especially, the hardening trend decrease up to the impact distance reaches the half of the beam. Instead, the increase of mass leads to an further hardening behaviour in all of case.
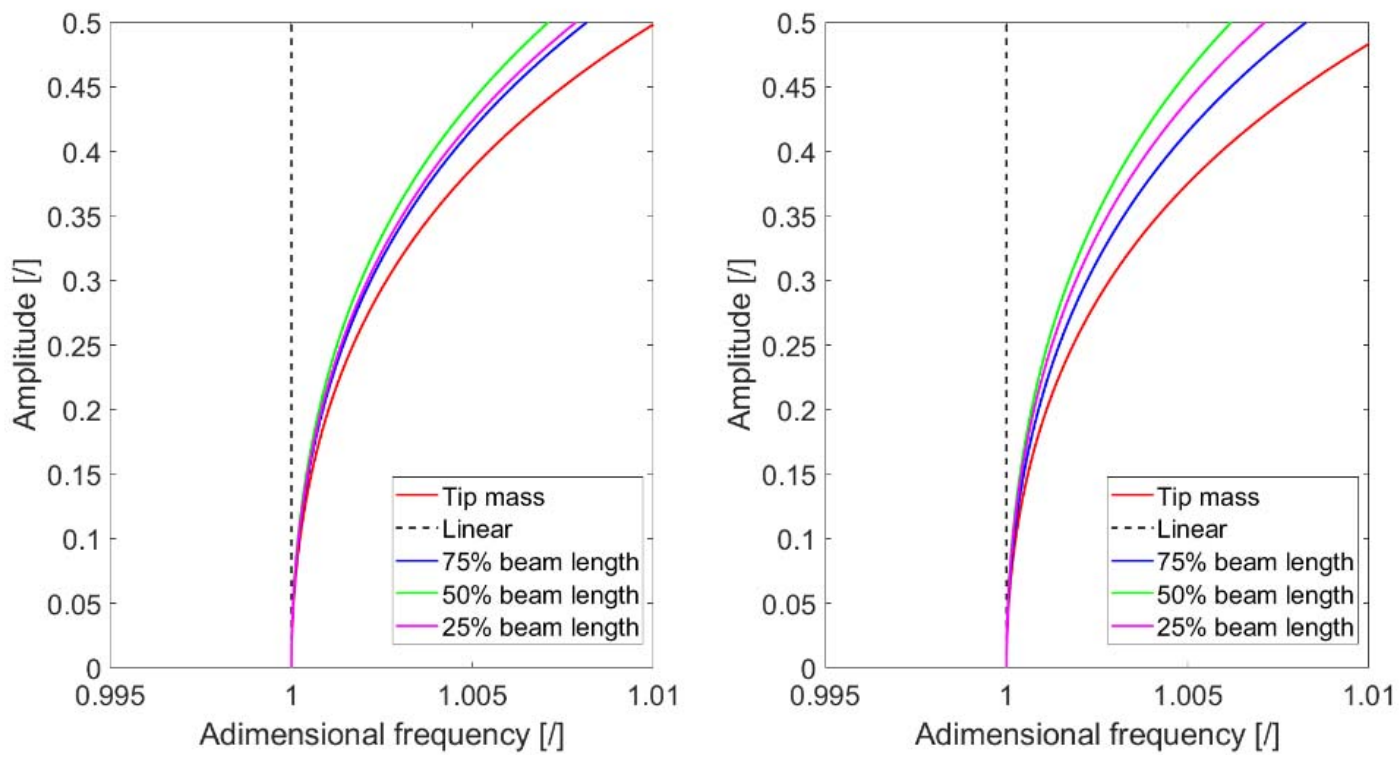

Figure 5: Backbone curve of first nonlinear frequency. (a) Influence of impacted distance $\eta$ of a projectile with a mass ratio $\mu$ of $50 \%$. (b)Influence of impacted distance $\eta$ of a projectile with a mass ratio $\mu$ of $200 \%$.

On the contrary the backbone curve of second and third modes are softening type, as shown in Fig. (6) and Fig. (7) respectively. Even in this cases were evaluated the influence of impacted distance $\eta$ of a projectile with a mass ratio $\mu$ of $50 \%$ and $200 \%$. The impacted distance $\eta$ changes the nonlinear trend.
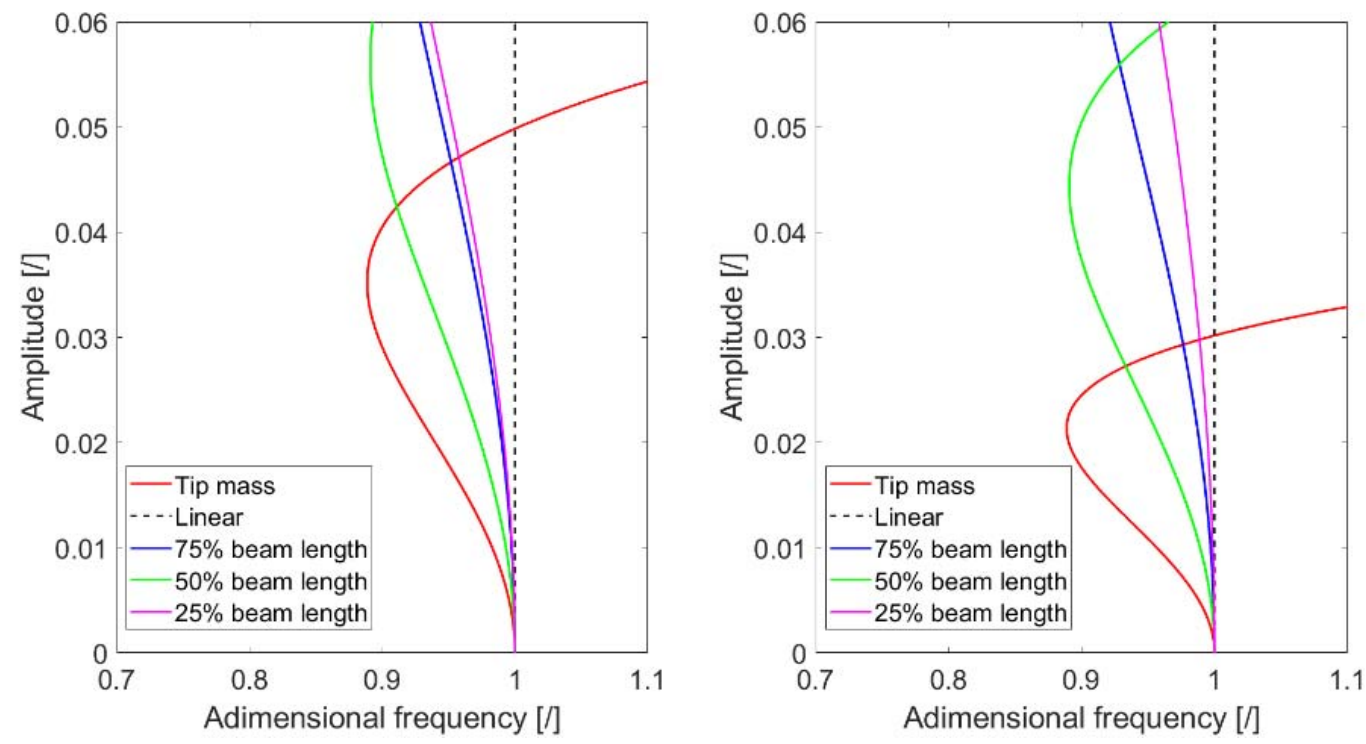

Figure 6: Backbone curve of second nonlinear frequency. (a) Influence of impacted distance $\eta$ of a projectile with a mass ratio $\mu$ of $50 \%$. (b)Influence of impacted distance $\eta$ of a projectile with a mass ratio $\mu$ of $200 \%$ 

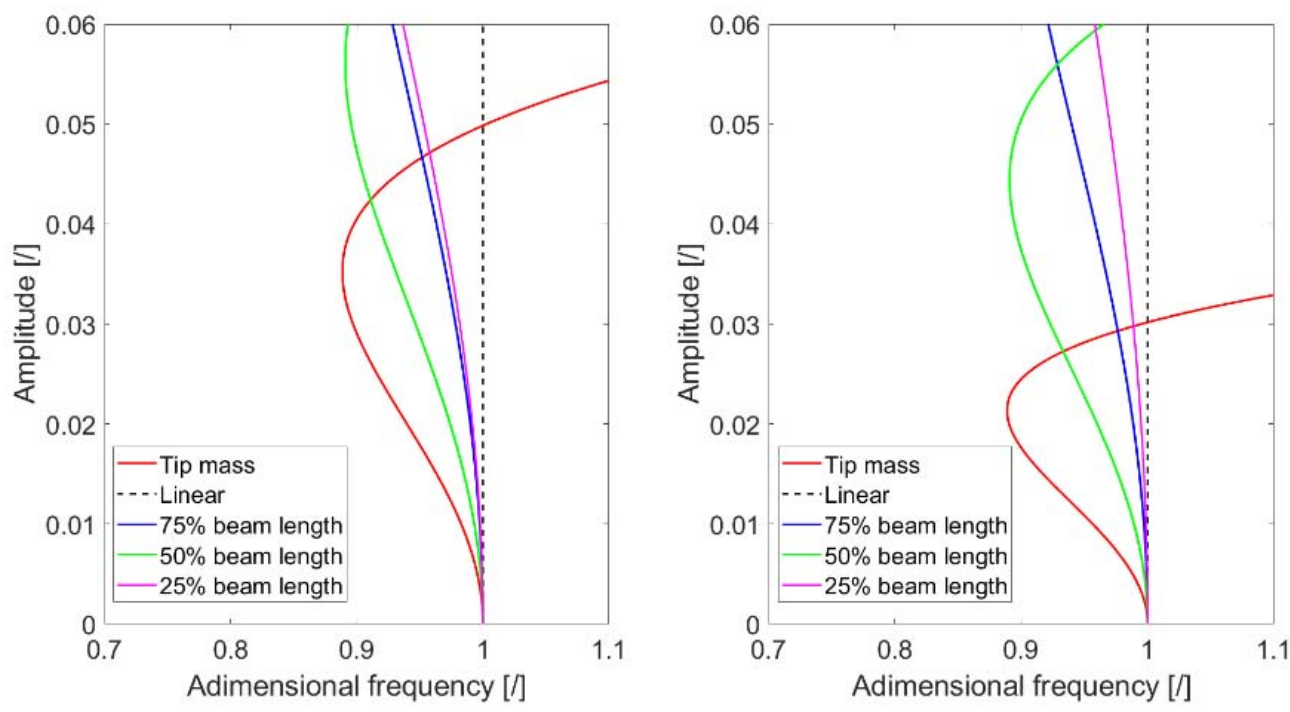

Figure 7: Backbone curve of third nonlinear frequency. (a) Influence of impacted distance $\eta$ of a projectile with a mass ratio $\mu$ of $50 \%$. (b)Influence of impacted distance $\eta$ of a projectile with a mass ratio $\mu$ of $200 \%$

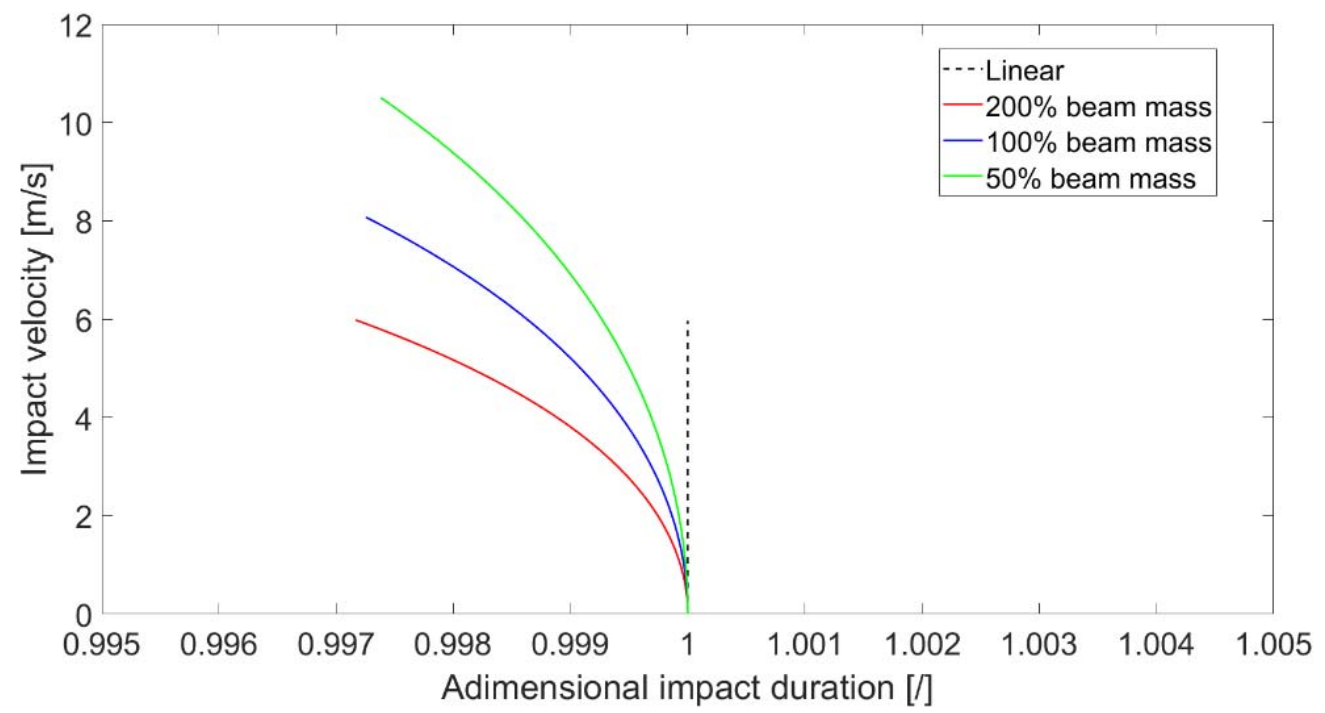

Figure 8: Trend of adimensional impact duration in function of mass ratio $\mu$. The impact occurs at $\eta=0.75$

The nonlinear behaviour of the frequency affects the response of the beam during the impacts. Following the spring-mass theory the projectile has the initial velocity and the beam is relaxed. Then, the projectile hits the beam and reduces its velocity up to the beam reaches the maximum deflection. The potential energy of the beam is equal to the initial kinetic energy of the projectile. After the maximum deflection point the projectile is pushed backward. The impact ends when the projectile overcomes the line of the clamped beam end. In large displacement the time of the impact can be reduced or augmented in function of the hardening or softening trend of nonlinear frequency. As the increase of the deflection of the beam this phenomenon becomes more and more relevant. The first nonlinear vibration is the most involved vibration when the impact occurs close to the free end of the beam. The amplitude due to the projectile impact with a velocity $v_{0}$ can be found through the Eqn.(31). The amplitude associated with the first modes increase as the velocity impact increasing. Therefore, the impact time is affected by the nonlinear frequency which is function of the amplitude. Starting from the Eqn.(24) and the assumption of first vibration response, the impact time is defined as 


$$
T_{\text {Imp }}=\frac{\pi}{\omega_{1 l}}=\frac{\pi}{\left(\omega_{10}+\omega_{12} A_{1}^{2}+\omega_{14} A_{1}^{4}\right)}
$$

Fig. (8) shows how the adimensional impact duration depends by the mass ratio $\mu$. Surely, the impact time is bigger as the increase of projectile mass. Moreover, the influence of nonlinearities is more relevant at high mass ratio with the same impact velocity. The explanation is related to the amplitude reached during the impact. The initial kinetic energy is strictly dependent to the mass ratio, so inevitably the beam has to deflect more at high mass ratio to absorb the impact at the same impact velocity. Likewise, can be evaluated the impact velocity in function of the maximum impact deflection. Starting from the Eqn.(31) and the assumption of first vibration response, the impact velocity can be computed as follows

$$
\begin{aligned}
& v_{0}=\frac{\int_{0}^{1} \Phi_{1}(\zeta)^{2} d \zeta}{\int_{0}^{1} \delta(\zeta-\eta) \Phi_{1}(\zeta) d \zeta} A_{1} \omega_{10} \text { linear, } \\
& v_{0}=\frac{\int_{0}^{1} \Phi_{1}(\zeta)^{2} d \zeta}{\int_{0}^{1} \delta(\zeta-\eta) \Phi_{1}(\zeta) d \zeta} A_{1}\left(\omega_{10}+\omega_{12} A_{1}^{2}+\omega_{14} A_{1}^{4}\right) \text { nonlinear }
\end{aligned}
$$
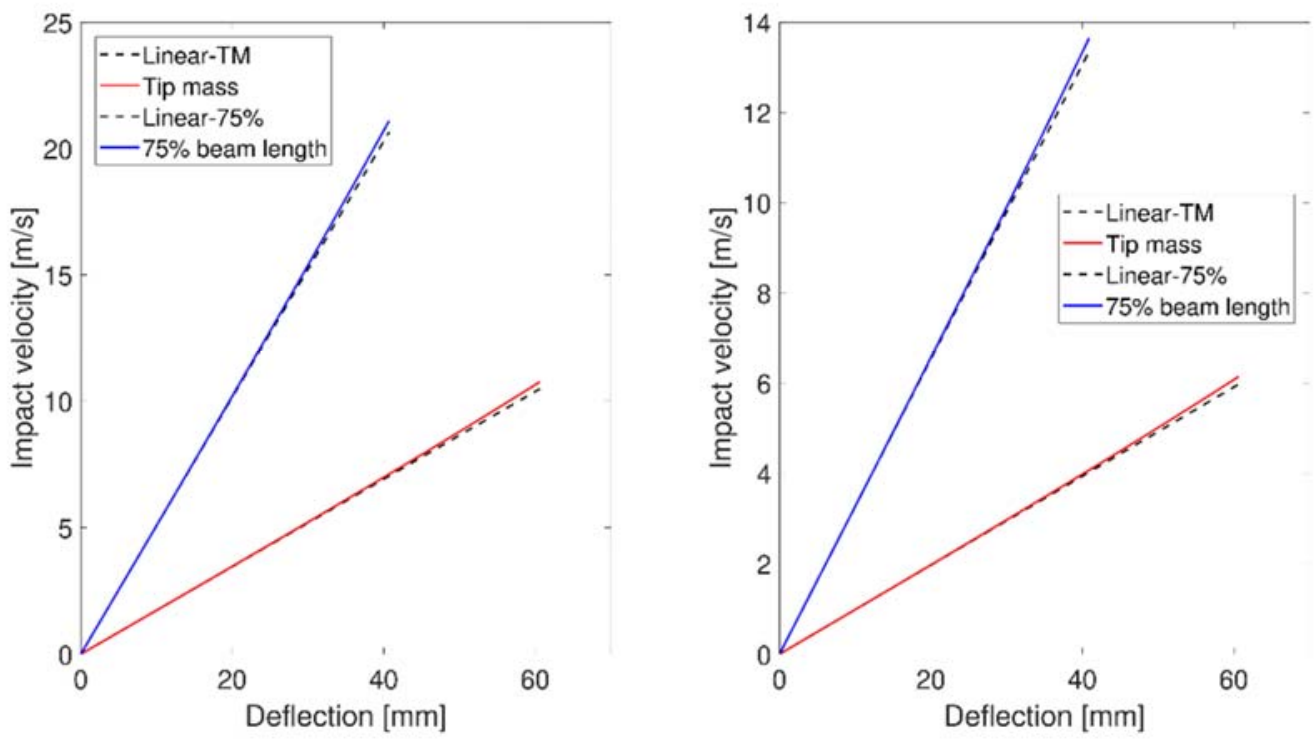

Figure 9: Impact velocity in function of the maximum deflection of the beam. (a) The projectile has a mass ratio $\mu$ of $50 \%$. (b) The projectile has a mass ratio $\mu$ of $200 \%$.

Fig. (9) shows how the impact velocity depends by the maximum deflection of the beam with two different mass ratio $\mu$. In large displacement the impact velocity is not linear but follow the nonlinear frequency trend. Therefore, the hardening behaviour of the first frequency leads to an increase of impact velocity to obtain the predicted amplitude in the linear theory. As the mass ratio increase the hardening behaviour persists and the nonlinear terms have more relevance, as shown in Fig. (9)b). The changing of the impact distance $\eta$ leads to an increase in slope of the impact velocity-amplitude relationship. Even in these case, the hardening behaviour persists but the nonlinear terms have less relevance. In impact dynamics the absorption energy on the impact is the key information as well as the displacements, and reactions forces. All information can be obtained by means of analytical model but the mono-modal approach is not able to describe the real time history of the system. For instance, the boundary conditions in Eqn.(30) impose the displacement is zero and the velocity is $v_{0}$ only in the position where the impact occurs. Taking into account only the first vibration mode is not possible to have this trend 
because the speed is function of only first mode shape. Following multi-modal superposition approach, a trend quite similar to the dirac function can be achieved. Therefore, a superposition of 10 modes is computed where only the first three modes takes into account nonlinear behaviour because of computational efforts. Fig. (10) shows the energy time history in a impact of projectile with $\eta=0.9, \mu=0.5$, and $v_{0}=5 \frac{\mathrm{m}}{\mathrm{s}}$.

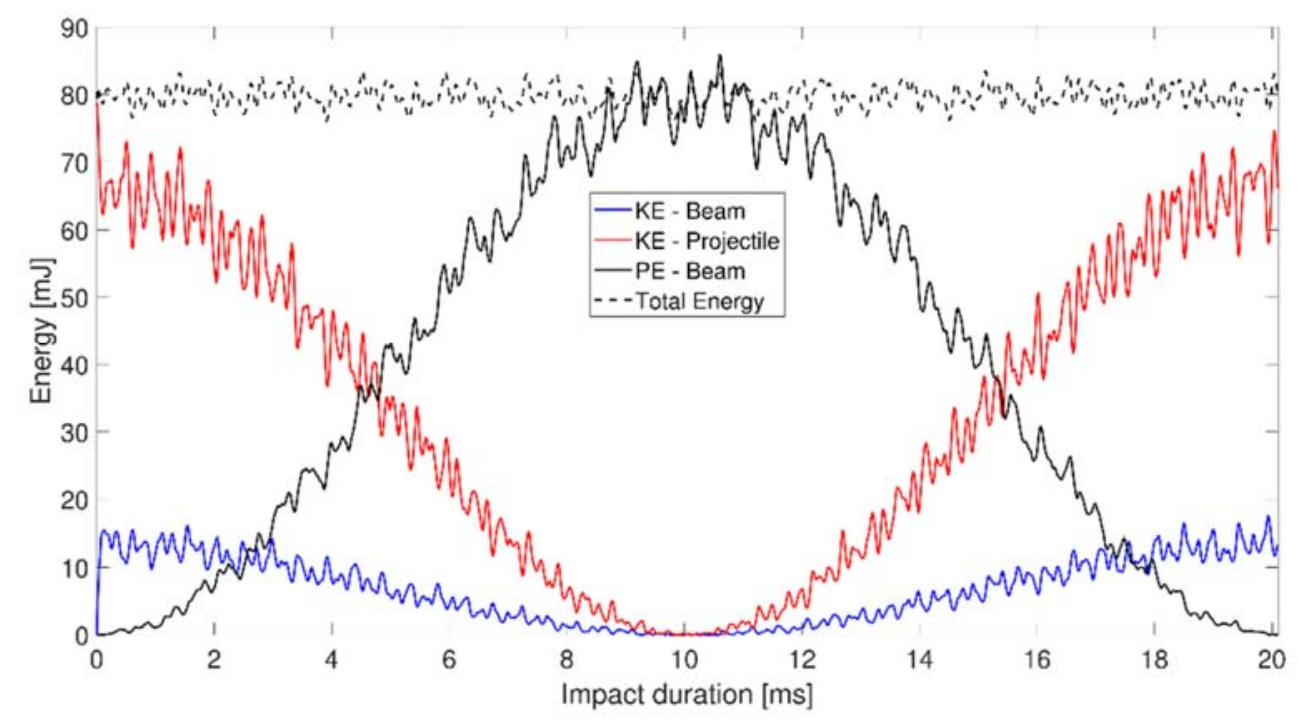

Figure 10: Energy time history.
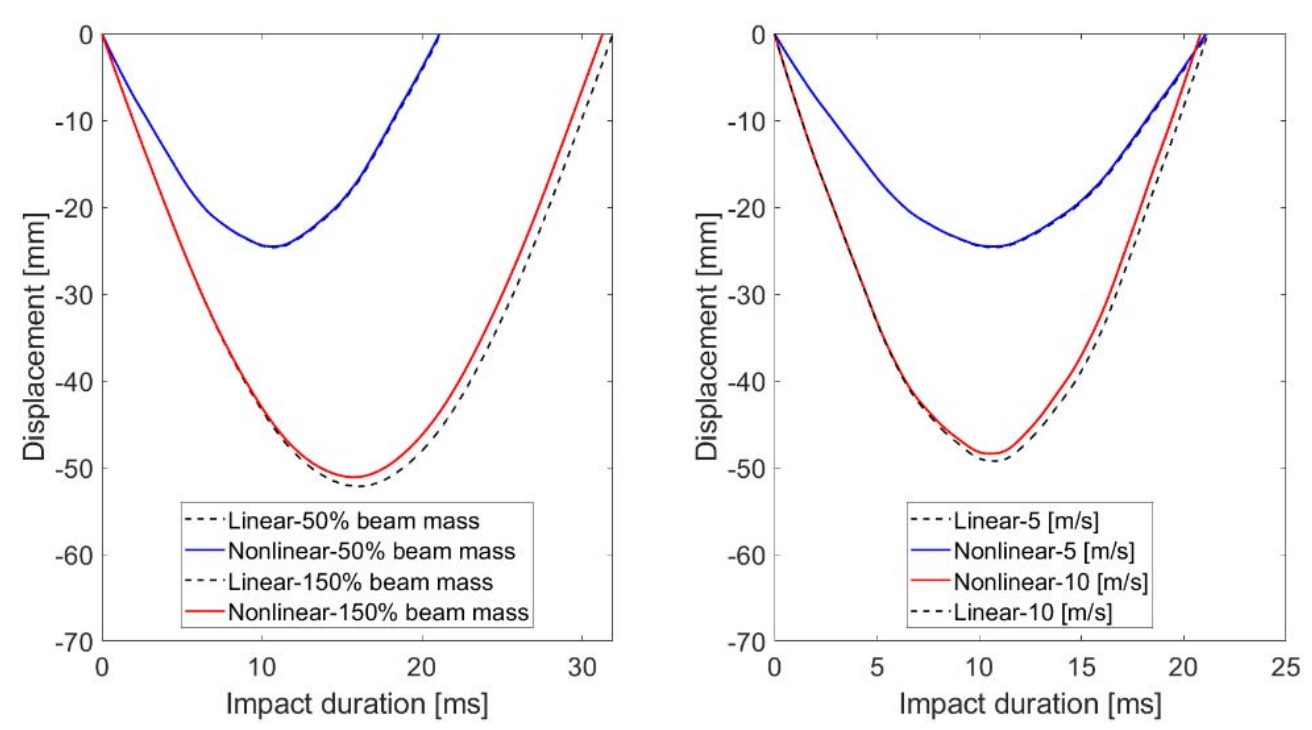

Figure 11: Deflection history of the free-end beam during the impact of projectile at $\eta=0.95$. (a) Influence of projectile mass with an initial speed of $5 \frac{\mathrm{m}}{\mathrm{s}}$. (b) Influence of projectile speed with a mass ratio $\mu$ of $50 \%$.

In order to confirm the validation of the model, the kinetic energy of the system is split between the beam and the projectile. At $t=0$ the kinetic energy of the projectile corresponds to the kinetic energy of free projectile with speed $v_{0}$. At the same time, the kinetic energy of the beam is very close to zero because it is relaxed. Increasing the numbers of modes into the model the beam reaches zero. In early stages, the beam undergoes the influence of the projectile velocity and starts to deflect. At the end of the impact, the beam continues to oscillate. The sum of the kinetic and potential energies represents the total energy of the system. The Fig. (10) shows the average of the total energy is constant. However, there are some fluctuations due to the numerical approximation in multi-modal approach and nonlinear equations. The effect of geometrical 
nonlinearities are noticeable in the displacement history as shown in Fig. (11). The displacement in linear and nonlinear response are quite similar in the trend. However, the time of impact is clearly reduced due to the hardening behaviour in the first nonlinear frequency. The effect also involves the peak of deflection. In the nonlinear model, the maximum amplitude reaches a lower amplitude than linear form. It is reduced because of the increase of stiffness in large displacement or rather the hardening behaviour in the first nonlinear frequency.

\section{Numerical Results}

First of all, the natural frequency obtained by the analytical model was compared with the numerical model. Tab.(2) shows this comparison in which some combination of $\eta$ and $\mu$ were set. The frequencies in the tables confirm the accuracy of the analytical model. The first frequencies are the best approximated by the analytical model. Instead the second and third frequencies are slightly greater than numerical ones.

\begin{tabular}{ccccc}
\hline$\eta$ & $\mu$ & $\begin{array}{c}\text { Mode } \\
\mathrm{n}\end{array}$ & $\begin{array}{c}\text { Analytical } \\
{[\mathrm{Hz}]}\end{array}$ & $\begin{array}{c}\text { FEM } \\
{[\mathrm{Hz}]}\end{array}$ \\
0 & 0 & $1^{\circ}$ & 39.333 & 39.331 \\
- & - & $2^{\circ}$ & 246.39 & 246.39 \\
- & - & $3^{\circ}$ & 690.20 & 690.20 \\
0.75 & 0.5 & $1^{\circ}$ & 28.771 & 28.770 \\
- & - & $2^{\circ}$ & 243.98 & 243.94 \\
- & - & $3^{\circ}$ & 583.38 & 582.65 \\
0.5 & 1 & $1^{\circ}$ & 32.328 & 32.326 \\
- & - & $2^{\circ}$ & 159.04 & 158.99 \\
- & - & $3^{\circ}$ & 690.03 & 689.30 \\
0.75 & 2 & $1^{\circ}$ & 18.575 & 18.572 \\
- & - & $2^{\circ}$ & 242.1731 & 242.01 \\
- & - & $3^{\circ}$ & 531.9391 & 531.21 \\
1 & 3 & $1^{\circ}$ & 11.697 & 11.697 \\
- & - & $2^{\circ}$ & 176.49 & 176.43 \\
- & - & $3^{\circ}$ & 563.31 & 562.80 \\
\hline
\end{tabular}

Table 2: Comparison of natural frequency between analytical and numerical model

The numerical analysis was carried out on the impact of a mass with $\mu=0.5$ at the impact distance $\eta=0.9$ with a initial velocity $v_{0}=5 \frac{\mathrm{m}}{\mathrm{s}}$. Firstly, the early stages of the impact was evaluated. The flexural waves start to travel inside the beam after the impact of the projectile. The flexural waves is showed in Fig. (12) at two instant of time, 0.00006 second and $0.00009 \mathrm{~s}$. In this time the flexural wave travels along to the beam and runs a distance of $40.58 \mathrm{~mm}, \Delta X_{\text {Wave }}$ in Fig. (12). The analytical flexural waves speed along the beam can be computed following the Eqn.(1). The impact occurs in a time about $0.00001 \mathrm{~s}$. Hence, the flexural wave speed is $1362.362 \frac{\mathrm{m}}{\mathrm{s}}$ and the theoretical $\Delta X_{\text {Wave }}$ is $40.87 \mathrm{~mm}$. Consequently, the flexural wave speed is verified. It is greater than the impact velocity and therefore the low-velocity assumption to model the impact is confirmed. After the early stages, the projectile and the beam start to motion following the multi-modal theory. In order to assess the compatibility of the analytical and numerical models, a comparison between the models results was evaluated. Fig. (13) shows the displacement and velocity histories of the impacted distance. The deflection computed with the analytical model overlaps the numerical ones, Fig. (13a). The impact time is perfectly described as well as the maximum deflection. Also, the analytical trend of velocity is consistent with the numerical one. However, the analytical signal appears smoother than the numerical solution, Fig. (13b). 


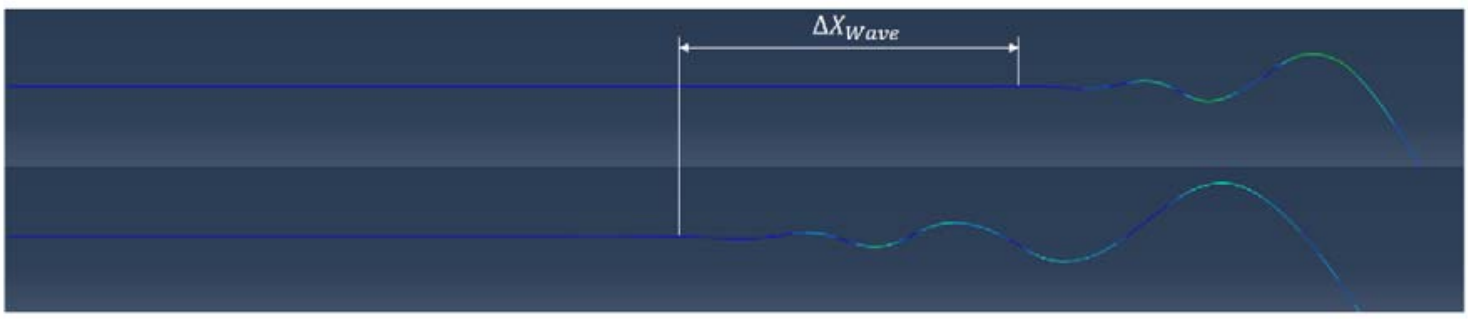

Figure 12: Flexural waves propagation in early stages of impact $(\Delta t=0.00003 s$ and scale factor 100$)$.
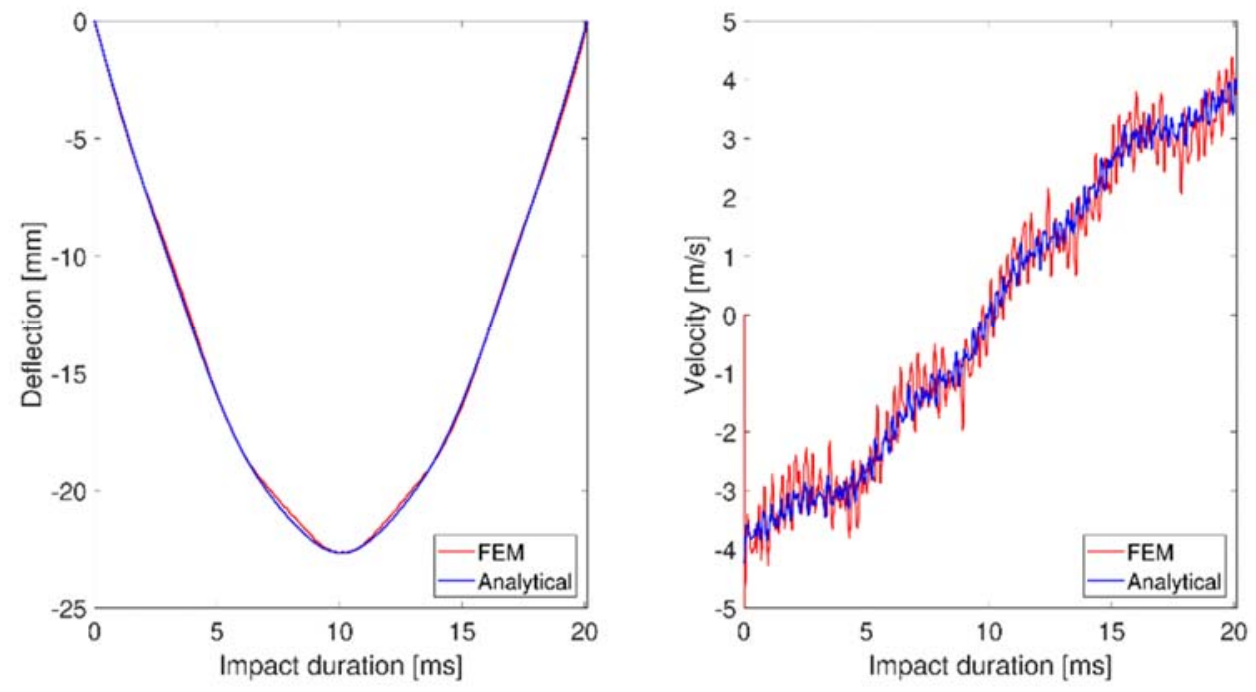

Figure 13: Impact of a mass with $\mu=0.5$ at the impact distance $\eta=0.9$ with a initial velocity $v_{0}=5 \frac{\mathrm{m}}{\mathrm{s}}$; Comparison of FEM and Analytical results.(a) Deflection history. (b) Velocity history

\section{CONCLUSION}

his paper investigated the nonlinear impact response in large displacements of a slender cantilever beam which undergoes the impact of a projectile. The spring-mass approach was used to describe the overall response and the indentation phenomenon was neglected because the overall deflection of the beam is much larger than local. The analysis compared the results coming from analytical approach with numerical model and experimental observations. As the nonlinear frequencies behaviour is function of the amplitude, the impact time is affected by the velocity impact. The hardening behaviour of the first frequency leads to a decrease of impact time as the velocity impact increase. Moreover, the hardening behaviour leads to an increase of impact velocity to obtain the same predicted amplitude in the linear theory. The experimental tests confirm the hardening behaviour of the first backbone curve. So, the analytical model was validated by the experimental findings. The numerical analysis also confirm the analytical outcomes. Consequently, the low-velocity assumption to model the impact is confirmed also in continuous beam hit by a projectile.

\section{REFERENCES}

[1] Beléndez T., Neipp C., Beléndez A., 2002. Large and small deflections of a cantilever beam. European Journal of Physics 23, 371. DOI: 10.1088/0143-0807/23/3/317. 
[2] Zheng Y., Shabana A. A., Zhang D., 2018. Curvature expressions for the large displacement analysis of planar beam motions. Journal of Computational and Nonlinear Dynamics 13. DOI: 10.1115/1.4037226.

[3] Abrate, S., 1998. Low-velocity impact damage, in: Impact on Composite Structures. Cambridge University Press, pp. 135-160. DOI: $10.1017 /$ cbo9780511574504.005.

[4] Abrate, S., 2001. Modeling of impacts on composite structures. Composite Structures 51, 129-138. DOI: 10.1016/s0263-8223(00)00138-0.

[5] Abrate, S., 2011. Impact Engineering of Composite Structures. Springer Vienna. DOI: 10.1007/978-3-7091-0523-8.

[6] Anderson T. J., Nayfeh A. H., Balachandran B., 1996. Experimental verification of the importance of the nonlinear curvature in the response of a cantilever beam. Journal of Vibration and Acoustics 118, 21-27. DOI: $10.1115 / 1.2889630$.

[7] Awrejcewwicz J., Kryosko A. V., Soldatov V., 2012. Analysis of the nonlinear dynamics of the timoshenko flexible beams using wavelets. Journal of Computational and Nonlinear Dynamics 7. DOI: 10.1115/1.4004376.

[8] Babilio E., Lenci S., 2017. On the notion of curvature and its mechanical meaning in a geometrically exact plane beam theory. International Journal of Mechanical Science 128-129, 277-293. DOI: 10.1016/j.ijmecsci.2017.03.031.

[9] Crespo da Silva M. R. M., Glynn C. C., 1978. Nonlinear flexural-torsional dynamics of inextensional beams II. Forced motions. Journal of Structural Mechanics 6, 449-461. DOI: 10.1080/03601217808907349.

[10] Eisley J. G., 1964. Nonlinear deformation of elastic beams, rings and strings. Wear 7, 225-312. DOI: 10.1016/00431648(64)90095-x.

[11] Goel, R.P., 1973. Vibrations of a beam carrying a concentrated mass. Journal of Applied Mechanics 40, 821-822. DOI: $10.1115 / 1.3423102$.

[12] Graff, K., 1991. Wave motion in elastic solids. Dover Publications, New York.

[13] Hamdan M. N., Dado M. H. F., 1997. Large amplitude free vibrations of a uniform cantilever beam carrying an intermediate lumped mass and rotary inertia. Journal of Sound and Vibration 206, 151-168. DOI: 10.1006/jsvi.1997.1081.

[14] Hinnant H. E., Hodges D. H., 1988. Nonlinear analysis of a cantilever beam. AIAA Journal 26, 1521-1537. DOI: $10.2514 / 3.10072$.

[15] Leissa, A., Sonalla, M., 1991. Vibrations of cantilever beams with various initial conditions. Journal of Sound and Vibration 150, 83-99. DOI: https://doi.org/10.1016/0022-460X(91)90403-7.

[16] Lenci, S., Clementi, F., 2019. Flexural wave propagation in infinite beams on a unilateral elastic foundation. Nonlinear Dynamics 99, 721-735. DOI: 10.1007/s11071-019-04944-4.

[17] Lenci S., Clementi F., Rega G., 2016. A comprehensive analysis of hardening/softening behavior of shearable planar beams with whatever axial boundary constraint. Meccanica 51, 2589-2606. DOI: doi.org/10.1007/s11012-016-0614-9.

[18] Lenci S., Consolini L., Clementi F., 2017a. The use of the Fitting Time Histories method to detect the nonlinear behaviour of laminated glass. Journal of Vibration Testing and System Dynamics 1, 1-14. DOI: 10.5890/jvtsd.2017.03.001.

[19] Lenci S., Consolini L., Clementi F., Cocchi G., 2017b. Revealing nonlinear dynamical behaviour of laminated glass. Procedia Engineering 199, 1454-1459. DOI: 10.1016/j.proeng.2017.09.394.

[20] Navaee S., Elling R. E., 1991. Large deflections of cantilever beams. Transactions of the Canadian Society for Mechanical Engineering 15, 91-107. DOI: 10.1139/tcsme-1991-0005.

[21] Nayfeh A. H., 2000. Perturbation methods. John Wiley \& Sons.

[22] Schwalbe D., Wagon S., 1997. The Duffing equation. John Wiley \& Sons.

[23] Hamdan M. N.and Shabaneh N. H., 1997. On the large amplitude free vibrations of a restrained uniform beam carrying an intermediate lumped mass. Journal of Sound and Vibration 199, 711-736. DOI: 10.1006/jsvi.1996.0672.

[24] Utzeri, M., Bhagavatam, A., Mancini, E., Dinda, G., Sasso, M., Newaz, G., 2021a. Quasi- Static and Dynamic Behavior of Inconel 625 Obtained by Laser Metal Deposition: Experimental Characterization and Constitutive Modeling. Journal of Engineering Materials and Technology 143. DOI: 10.1115/1.4051087.

[25] Utzeri, M., Farotti, E., Coccia, M., Mancini, E., Sasso, M., 2021b. High strain rate compression behaviour of 3d printed carbon-PA. Journal of Materials Research DOI: 10.1557/s43578-021-00248-9.

[26] Utzeri, M., Sasso, M., Chiappini, G., Lenci, S., 2020. Nonlinear Vibrations of a Composite Beam in Large Displacements: Analytical, Numerical, and Experimental Approaches. Journal of Computational and Nonlinear Dynamics 16. DOI: 10.1115/1.4048913.

[27] Wagner H., 1964. Large-amplitude free vibrations of a beam. Journal of Applied Mechanics 32, 887-892. DOI: $10.1115 / 1.3627331$. 
[28] Warminski, J., Kloda, L., Lenci, S., 2020. Nonlinear vibrations of an extensional beam with tip mass in slewing motion. Meccanica 55, 2311-2335. DOI: 10.1007/s11012-020-01236-9.

[29] Zavodney L. D., Nayfeh A. H., 1989. The non-linear response of a slender beam carrying a lumped mass to a principal parametric excitation: theory and experiment. International Journal of Non-Linear Mechanics 24, 105-125. DOI: 10.1016/0020-7462(89)90003-6. 